\title{
Kernos
}

Revue internationale et pluridisciplinaire de religion

grecque antique

29 | 2016

Varia

\section{The Greek Gods in Modern Scholarship.}

Interpretation and Belief in Nineteenth and Early

Twentieth Century Germany and Britain

\section{Giuseppina Paola Viscardi}

\section{Q OpenEdition}

12 Journals

\section{Édition électronique}

URL : http://journals.openedition.org/kernos/2451

DOI : $10.4000 /$ kernos. 2451

ISSN : 2034-7871

Éditeur

Centre international d'étude de la religion grecque antique

\section{Édition imprimée}

Date de publication : 1 octobre 2016

Pagination : 471-475

ISSN : 0776-3824

\section{Référence électronique}

Giuseppina Paola Viscardi, «The Greek Gods in Modern Scholarship. Interpretation and Belief in Nineteenth and Early Twentieth Century Germany and Britain », Kernos [En ligne], 29 | 2016, mis en ligne le 01 octobre 2016, consulté le 19 novembre 2020. URL : http://journals.openedition.org/kernos/ 2451 ; DOI : https://doi.org/10.4000/kernos.2451

Ce document a été généré automatiquement le 19 novembre 2020.

Kernos 


\title{
The Greek Gods in Modern Scholarship. Interpretation and Belief in Nineteenth and Early Twentieth Century Germany and Britain
}

\author{
Giuseppina Paola Viscardi
}

\section{RÉFÉRENCE}

Michael D. KONARIS, The Greek Gods in Modern Scholarship. Interpretation and Belief in Nineteenth and Early Twentieth Century Germany and Britain, Oxford Classical Monographs, Oxford, Oxford University Press, 2016. 1 vol. $14 \times 22 \mathrm{~cm}, \mathrm{x}+361$ p. ISBN :

978-0-19-873789-6.

1 Le livre de Michael D. Konaris (M.D.K.) offre un panorama précis de l'histoire des études et de l'interprétation dans une perspective historico-religieuse du polythéisme grec, concentrant son analyse sur la période comprise entre le $\mathrm{xIX}^{\mathrm{e}} \mathrm{s}$. - date à laquelle on place conventionnellement l'apparition de la science des religions moderne, liée principalement aux noms de F. Max Müller, Edward B. Tylor, James G. Frazer ${ }^{1}-$ et le début $d u x^{e}$ s., en privilégiant les lignes de recherche développées principalement par les écoles allemandes et britanniques. De cette étude naît un tableau articulé et raisonné des principales visions théoriques et des approches méthodologiques qui ont dominé l'histoire du philhellénisme tant allemand que victorien, encadré dans une description plus générale du panorama culturel, historique et religieux des deux Étatsnation à leurs débuts.

2 La recherche de M.D.K. se fonde sur le travail de doctorat présenté au Balliol College d'Oxford sous la direction de Robert Parker (dont on sent bien l'influence sur la 
méthodologie), Oswyn Murray et Scott Scullion; elle se développe à l'Institut für Religionswissenschaft de la Freie Universität de Berlin, où l'A. à participé au projet dirigé par Renate Schlesier «Der differente Gott. Konstruktionen des Dionysos in der Moderne", dans une ambiance stimulante de dialogue interdisciplinaire, riche en expériences sur l'étude des interprétations modernes de la religion grecque.

L'histoire de la science moderne de la religion grecque est un domaine de recherche qui attire toujours plus l'attention de ceux qui s'occupent de la Grèce antique, mais aussi de quiconque s'intéresse plus généralemen à l'histoire moderne des idées, de la culture et de la religion. De ce point de vue, le livre commence par affirmer que «il n'y a pas de vision finale des dieux grecs » : notre vision des dieux grecs, exactement comme celle de nos prédécesseurs, est nécessairement ancrée et idéologiquement conditionnée par les lieux et les époques de leur production, les «situations und zeibedingt » d'Albert Henrichs. Le choix de centrer l'attention sur une période précise et sur une partie de la recherche à caractère national, tout en rendant compte de l'histoire des études en d'autres contextes (cf. les travaux de Renan, Maury, de Coulanges, juste pour rester dans un contexte francophone), est motivé par l'idée de base que «certains, parmi les développements les plus importants dans l'étude de la religion grecque durant le XIXe et le début du xx $\mathrm{x}^{\mathrm{e}}$ siècles, sont advenus en Allemagne, la source de la Altertumswissenschaft, et au Royaume-Uni, le centre de la Darwinian anthropology » (p. 2-3, Introduction).

Les spéculations théoriques sur l'interprétation des divinités grecques nées dans chacun des contextes de la philologie, de l'archéologie, de la mythologie comparée, de la sociologie ou de l'anthropologie, ne relèvent pas d'un simple intérêt d'antiquaire, mais font partie d'un débat moderne plus ample sur la religion, sur le rapport entre l'homme et la nature, le monde classique et le christianisme, la culture occidentale et orientale. L'étude de la religion et du panthéon grecs s'est déroulée avec, en arrièreplan, les débats entre les partisans et les détracteurs de l'athéisme scientifique, les rivalités entre catholiques et protestants, les différences idéologiques et culturelles entre les Anglais et les Allemands, ce qui a conditionné l'interprétation et a donné à cette science une valeur paradigmatique pour le présent. L'enquête sur les modalités de développement d'un tel champ d'études en relation avec son contexte historique de production nous fournit une opportunité de réflexion sur l'état actuel de la recherche et sur les thèmes principaux qui animent les débats contemporains sur la nature, les aspects, les fonctions et les champs d'action des différents dieux grecs, outre que sur le fonctionnement structuré - ou plutôt a-systématique - du polythéisme antique.

Comme le précise l'A., dans l'ample introduction du livre (p. 1-51), l'étude se concentre sur deux verrous qui ont animé le débat moderne au lendemain de la polémique qui a suivi la publication du travail de G.F. Creuzer, Symbolik und Mythologie der alten Völker

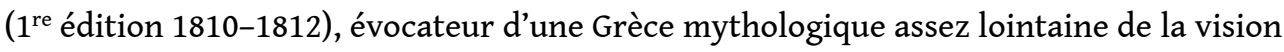
néoclassique du siècle précédent. En ligne avec la tendance qui vise à attribuer aux origines du mythe une valeur interprétative très élevée, qui a dominé les recherches du $\mathrm{XVIII}^{\mathrm{e}} \mathrm{s}$., de B. de Fontenelle à C. Dupuis, les questions prises en compte au siècle suivant ont touché, d'un côté, la nature de la religion grecque - c'est-à-dire la question de savoir si elle était originellement monothéiste ou polythéiste - et, de l'autre, les origines et la dynamique entre les principales divinités du panthéon hellénique, avec une attention particulière portée à la figure d'Apollon - dans le sillage des interprétations des premiers symbolistes à la Dupuis (De l'Origine des Tous les Cultes, 1795) ou à la C.G. Heyne, considéré comme le fondateur de la science moderne de la 
mythologie comparée en Allemagne, les deux héritiers à leur tour de la leçon de Macrobe - Apollon comme une incarnation directe du dieu Soleil, selon une conception qui remonte aux ancienne doctrines sacerdotales orientales ${ }^{2}$.

Durant les $\mathrm{XVIII}^{\mathrm{e}}$ et $\mathrm{XIX}^{\mathrm{e}} \mathrm{s}$., le débat moderne sur les origines de la religion a été marqué par la nécessité d'établir une fois pour toutes si la religion originelle de l'humanité était monothéiste ou polythéiste. En conséquence, on a observé une tendance à identifier des éléments polythéistes dans les grand monothéismes et, vice versa, des éléments monothéistes dans les religions païennes. S'est aussi développée l'idée d'un processus avançant par étapes, passant graduellement du polythéisme au monothéisme, selon la théorie de E.B. Tylor (Primitive Culture, 1871), qui remonte au positivisme comtien (Cours de philosophie positive, 1869), ou avant cela aux réflexions de D. Hume (The Natural History of Religion, 1757) et de J.-J. Rousseau (Émile, 1762). À cette idée s'opposaient le concept d' " autonomie génétique », développé par A. Lang (The Making of Religion, 1898), et de "monothéisme primordial» (Urmonotheismus), antérieur à toute autre forme de religion tel que formulé par P.W. Schmidt (Der Ursprung der Gottesidee, I, 1912) à partir de la conception rationaliste de Voltaire (Mélanges, 1761 et Dictionnaire philosophique, 1764) - propagateur des idées déistes en France - et de la théologie remontant aux Pères de l'Église ${ }^{3}$.

7 Malgré les polémiques et les critiques, l'Urmonotheismus est resté une orientation prédominante dans les études religieuses au XVIII ${ }^{e}$ et surtout au $\mathrm{XIX}^{\mathrm{e}} \mathrm{s}$. On retrouve un écho d'une telle orientation dans les recherches sur la religion grecque, et en premier lieu dans l'œuvre du théologien français N.S. Bergier (L'Origine des Dieux du Paganisme, 1774), qui sera ensuite reprise par F.G. Welcker (Griechische Götterlehre, 1857-1863) et par son disciple E. Curtius (Griechische Geschichte 1, 1857 ; Alterthum und Gegenwart, 1875) dans le cadre des études philologiques germaniques. E. Curtius a été à l'origine de la théorie des dieux universels - dans la ligne d'une tradition que remonte, à travers K.O. Müller, à la théologie romantique de F. Schleiermacher - à laquelle s'opposera avec sa théorie des Sondergötter (Götternamen, 1896) l'autre fameux disciple de Welcker, H. Usener. Welker mettait l'accent sur la transition des dieux grecs qui passaient du statut de divinités naturelles à celui de divinités personnelles ou « éthiques » en accord avec la vision chrétienne et le sentiment romantique de la nature caractéristiques de l'œuvre de P.W. Forchhammer (de Hellenika, 1837, à Homer, 1893), en considérant aux origines de la religion grecque soit l'idée d'une divinité singulière (un Urgott suprême incarné par Zeus Kronios) que un Naturdienst polythéiste.

Bien que ce fût contraire tant à la vision par Welcker d'un Urmonotheismus grec (et en général au dogme de une Urreligion monothéiste enraciné dans les études germaniques) qu'à la théorie selon laquelle le polythéisme grec serait le produit final de l'unification de divers cultes tribaux (une théorie avancée par son maître K.O. Müller - le représentant majeur de l'approche historico-critique de la religion grecque qui s'est opposée pendant une bonne partie du $\mathrm{XIX}^{\mathrm{e}} \mathrm{s}$. aux interprétations naturalistes de l'école germanique - et poursuivie par Heinrich Dietrich Müller), L. Preller ne renonça pas à voir des éléments monothéistes au sein du polythéisme grec (Griechische Mythologie, 1854-1855). Parmi les arguments en faveur de la nature plutôt monothéiste de la religion grecque, on trouve la tendance à transformer l'esprit du ciel en la figure hégémonique du régulateur du monde, qui touchait son point final dans la figure du Zeus d'Eschyle qui était célébré dans l'hymne à Zeus de l'Agamemnon, comme le plus haut, le plus sage et le plus bienveillant parmi les dieux; quitte à regrouper toute la 
multitude des manifestions divines présentes dans le mythe, dans le culte, dans l'art, de manière à suggérer une unité de fond du monde divin, mise en évidence, outre Preller, par C.F. Nägelsbach (Homerische Theologie, 1840) et ensuite par E. Gerhard (Griechenlands Volkstämme und Stammgottheiten, 1854).

On a connu une reprise - bien que dans certaines limites - des positions théoriques assumées par les philologues, les historiens et les archéologues allemands mentionnés plus haut dans le domaine des recherches anthropologiques de l'école britannique, avec L.R. Farnell (Cults of the Greek States, I, 1896). Ce dernier, en polémique avec son mentor A. Lang (Myth, Ritual and Religion, 1887, The Making of Religion, 1898), avait tendance à voir - tout comme Preller et Welcker - dans la tendance au regroupement et à la classification du culte des figures divines, la preuve d'une attitude monothéiste ou, plus précisément, énothéiste de la religion grecque en interprétant cette attitude comme le résultat d'une progression de la pensée religieuse plutôt que comme le résidu de l' Urmonotheismus grec. Cependant, à différence de Preller, Farnell était bien loin de soutenir que Zeus serait le point central du polythéisme grec, tout en lui reconnaissant une suprématie sur les autres divinités du panthéon ; de même, Farnell n'acceptait pas que la conception monothéiste de la divinité - une conception identifiable dans la pensée de poètes et philosophes comme Eschyle et Cléanthe - ait conditionné les formes de religiosité populaires.

10 En parallèle aux tentatives de médiation entre les vieilles et nouvelles orientations de la science par Farnell à Oxford, une réponse plus révolutionnaire au nouveau contexte d'étude révélé par l'émergence de disciplines comme l'anthropologie et la sociologie est venue de J.E. Harrison (Prolegomena to the Study of Greek Religion, 1903; Themis: A Study of the Social origins of Greek Religion, 1912) et des changements méthodologiques introduits dans la science religions et dans les études classiques par l'influence frazerienne du groupe dit des "Ritualistes de Cambridge", comme F.M. Conford, A.B.Cook ou l'oxonien G. Murray. Dans son analyse sociale des origines de la religion, Harrison reprenait l'approche darwinienne de l'anthropologie de Tylor et adoptait une perspective qui mettait en discussion la centralité des dieux de l'olympe dans le panthéon grec, sur la lignée tracée par les recherches de E. Rohde (Psyche, 1890, 1894). Harrison redécouvrait aussi l'agency des dieux chthoniens, qu'elle entendait comme des divinités locales dont le culte constituait la principale caractéristique du paysage religieux post-homérique, ce qui renforçait l'idée que les poèmes homériques ne représentaient pas le point initial de la mythologie et que la religion grecque, par analogie avec le living religions, n'avait pas été conçue comme un cosmos olympien ordonné, mais comme un conglomérat «fluide» de cultes à plusieurs niveaux, partiellement en conflit avec des variantes locales. L'emphase mise par Harrison sur les pratiques rituelles et sur les aspects sociaux de l'expérience religieuse (l'initiation en particulier) a marqué l'étude de la religion grecque pendant tout le $\mathrm{xx}^{\mathrm{e}} \mathrm{s}$. jusqu'à nos jours, apposant son empreinte sur les travaux de L. Gernet et H. Jeanmaire et, plus tard, sur ceux de J.-P. Vernant et W. Burkert (p. 265, Chap. 4).

11 En faisant appel à l'idée de Croce selon laquelle "l'histoire est toujours contemporaine " (cf. La storia come pensiero e come azione, Bari, 1938), une idée reprise par R.G. Collingwood (The Idea of History, New York, 1946) et importée dans les études post-modernes actuelles, le travail de M.D.K. met une fois de plus l'accent sur l'alliance étroite, remontant à la fin du $\mathrm{XIX}^{\mathrm{e}}$ et au début $d u \mathrm{Xx}^{\mathrm{e}} \mathrm{s}$., entre l'interprétation du panthéon grec et la recherche sur les principales questions contemporaines à caractère 
religieux et culturel : «Interprétés comme dieux aux caractères spécifiques ou dieux universels, comme descendants de Sondergötter ou Totems, comme personnalités éthiques idéales ou projections d'émotions collectives, les dieux grecs témoignent de l'existence de différentes croyances, intérêts et intentions modernes. Les théories en compétition qui apparaissent dans les études nous permettent de discerner comment la science de la religion grecque s'investit significativement dans le présent et donne de forts exemples de l'intersection entre l'histoire des études classiques et celle de la culture, de la théologie et de l'intellectualité modernes» (p. 266, Conclusion). Le livre nous offre un bon exemple de la capacité de l'A. à mettre en contexte et à identifier les questions relevant des thèmes et des méthodes de l'analyse historico-religieuse, tout en mettant en évidence les problématiques herméneutiques et les défis épistémologiques dans le panorama culturel de l'Europe moderne. Il nous fournit ainsi un instrument de recherche valide au service des chercheurs en religion grecque ou, plus généralement, en histoire de la culture et des idées.

\section{NOTES}

1. .Sur ce sujet, nous rappelons le dernier travail de G.G. STRoumSA, A New Science: The Discovery of Religion in the Age of Reason, Cambridge (Mass.), 2010, où le spécialiste des religions abrahamiques et de l'étude comparée des religions propose de (re)dater la naissance de la science moderne des religions à une époque très antérieure, comprise entre le $\mathrm{XVII}^{\mathrm{e}}$ et le XIX ${ }^{\mathrm{e}}$ siècle, à cheval entre la Renaissance et le Romantisme, quand «un nouveau paradigme de la religion était crée, un paradigme qui permettait une révolution intellectuelle » (p. vIII).

2. .En appendice au volume (p. 289-298), l'A. revient sur la figure d'Apollon comme un cas prototypique des études classiques, en reprenant du point de vue théorique une récente contribution sur la figure d'Apollon et ses origines dans l'historiographie du XIX $\mathrm{e}$ s., où il a occupé une place prépondérante, notamment à la suite de l'œuvre de K.O. Müller (Die Dorier, 1824). Ce dernier a été le premier à supposer une origine grecque du dieu, et plus particulièrement dorienne, en opposition à l'origine " orientale » qui était communément acceptée avant lui, mais sa thèse ne sera guère suivie dans la réception de son œuvre. Cf. M.D. KonARIS, "Apollo in Nineteenth-Century Scholarship: The Case of K.O. Müller », in M. HAYSoM, J. WALLENSTEN (éd.), Current Approaches to Religion in Ancient Greece, Stockholm, 2011, p. 13-22.

3. .Une lecture semblable a été proposée dans le contexte du panorama des études d'histoire des religions en Italie : cf. R. PetTAzzonI, Prefazione ai Saggi di storia elle religion e di mitologia, Rome, 1946, nouvelle édition par G. Casadio, Naples, 2013, p. 9-20. 


\section{AUTEURS}

\section{GIUSEPPINA PAOLA VISCARDI}

Università degli Studi di Salerno 